\title{
Deep Learning-based Mammogram Classification for Breast Cancer
}

\author{
Gokhan ALTAN ${ }^{1}$
}

\section{Submitted: 19/09/2020 Accepted : 04/12/2020}

\begin{abstract}
Deep Learning (DL) is a rising field of researches in last decade by exposing a hybrid analysis procedure including advanced level image processing and many efficient supervised classifiers. Robustness of the DL algorithms to the big data enhances the analysis capabilities of machine learning models by feature learning on heterogeneous image database. In this paper, Convolutional Neural Network (CNN) architecture was proposed on simplified feature learning and fine-tuned classifier model to separate cancer-normal cases on mammograms. Breast Cancer is a prevalent and mortal disease appeared resultant mutating of normal tissue into tumor pathology. Mammograms are the common and effective tools for the diagnosis of breast cancer. DL-based computer-assisted systems have capability of detailed analysis for even small pathology that may lead the curing progress for a complete assessment. The proposed DLbased model aimed at assessing the applicability of various feature-learning models and enhancing the learning capacity of the DL models for an operative breast cancer diagnosis using CNN. The mammograms were fed into the DL to evaluate the classification performances in accordance with various $\mathrm{CNN}$ architectures. The proposed Deep model achieved high classification performance rates of $92.84 \%, 95.30 \%$, and $96.72 \%$ for accuracy, sensitivity, specificity, and precision, respectively.
\end{abstract}

\section{Keywords: Deep Learning, Convolutional Neural Networks, Breast Cancer, Mammogram, DDSM, Transfer Learning}

\section{Introduction}

Breast cancer is a common disease with high mortality rate for female in particular. Though the breast cancer patient population consists phenomenal male, the majority of 1.5 million deaths per year possesses female population [1]. Like many types of cancer, early diagnosis of breast cancer is important to reduce the mortality of the disease. Hence, the governments carry out very intensive studies to start treatment by making diagnosis especially at an early stage, and transfers mobile diagnostic systems to countryside. They foresee the managing certain physical examinations for women population in the risk group. Despite the health policies, the mortality rate could not nonetheless be reduced to desired levels. In this case, the switching on computerassisted systems, which have capable of service in a more widespread environment as diagnostics, is important and necessary. Mammograms are the most common diagnostics to visualize the cancer pathology using low-dose x-ray [2]. Since permeability of cancer lesion is less than healthy tissue, it renders white pathology on the mammograms. Therefore, asymptotic expansion of breast cancer at severity stages can be identified and monitored using mammograms, as well. Detecting cancer lesion in the early stage is time-consuming and compulsive progress for even experienced physicians on account of mammograms are noisy grey-scale images [3, 4]. Moreover, this procedure composes necessity to additional clinical tests, workload, doublereading, and more [5]. Early diagnosis of breast cancer can be supported using computer-assisted diagnostic (CADx) methods for starting the treatment before spreading too many tissues. The CADx systems can analyse the whole mammogram with advanced techniques to detect small cancer lesions and can speed

${ }^{1}$ Computer Eng., Iskenderun Technical University Hatay, TURKEY ORCID ID: 0000-0001-7883-3131

*Corresponding Author Email: gokhan.altan@iste.edu.tr up treatment and precautions for progression. Conventional CADx systems on breast cancer need pre-processing, pattern, histogram, handcrafted and shape-based features [6]. Whereas medical analysis methods focus on extracting handcrafted features, decomposition procedures, and expert guidance [7, 8], novel CADx systems perform hybrid analysis for identification of cancer pathology and application of learning procedures on cancer-specific lesions.

Deep Learning (DL), which is a prosperous classifier on image analysis, is at the leading place at the best ranking list of machine learning algorithms due to feature learning and transfer learning. DL impairs the necessity of extracting handcrafted features using advanced level image processing techniques by transferring the feature activation maps for next convolution layers. At the last convolution layer, it flattens the significant pixel matrix into the multi-layered fully connected layers to learn these features in supervised ways $[9,10]$. The convolution capacity of the proposals led to name the algorithm as Convolutional Neural Networks (CNN). The greatest convenient of the CNN is autoextracting the various level features from input images by feature learning and establishing models with many hidden layers and big number of neurons at each hidden layer $[10,11]$.

In recent years, a majority of researches applied DL to many image types including images, radiography images, satellite images, time-series, and more. DL has novel papers on analysis of mammograms for cancer diagnosis, identification of calcification and segmentation of cancer lesions by re-training of popular pre-trained $\mathrm{CNN}$ architectures including AlexNet, DenseNet, VGGNet, GoogleNet, DenseNet on different datasets [4, 12-15]. They achieved high classification performances for breast cancer. However, the big number of classification parameters $(10 \mathrm{M} \sim 132 \mathrm{M})$ is still the most unfavourable characteristic of pre-trained CNN models [14-16].

Conventional approaches on cancer detection focused on handcrafted features using advanced image processing techniques and 
pre-processing [17]. Yoon and Kim used handcrafted features including mass shape, mass margin, and calcification type to classify mammograms into benign and malignant. They fed the feature set into the support vector machines (SVM) with nonlinear radial basis function an reported high training accuracy for AdaBoost and SVM [17]. Sarosa et al. used region of interests (ROI) to detect malignant and benign masses in mammograms for breast cancer. They extracted handcrafted features including contrast sharpening, histogram, and grey-level co-occurrence matrix. They achieved high classification performances by feeding the hand-crafted feature set into the SVM classifier [7]. Novel approaches, such as CNN, provide layer-by-layer analysis, feature learning, and transfer learning on classification of mammograms for breast cancer on medical images [6, 8]. Pardamean et al. classified the mammograms as normal and cancer. They analysed mammograms using pre-training of ChexNet and DenseNet models and experimented on the impact of the various classification parameters including learning rate, dropout factor and L2 regularization [19]. Ertosun and Rubin established a model to classify mammograms into mass and normal for breast cancer on augmented mammograms. They retrained the pre-trained $\mathrm{CNN}$ architectures and highlighted the efficiency of GoogleNet on mammography images [20]. Xi et al. analysed class-based activation maps on pre-trained CNN architectures for separation of calcification and mass on mammograms. They assessed the mass detection performances of AlexNet, VGGNet, GoogleNet, and ResNet architectures and reported the highest achievements from VGGNet for detecting the cancer lesions [18]. Most of the researchers analysed the ROI segments instead of using whole mammograms to detect the mass and cancer lesions. Zeng et al. enhanced the CNN using a smoothing technique during convolution and the ROI pooling for mass detection. They focused on shape-based combinations of mass on mammograms to identify the breast cancer [3]. Suzuki et al. proposed a CADx system to separate the mammograms into mass and normal using ROIs. They utilized transfer learning feature form AlexNet architecture [21]. Nguyen and Lim adapted Gabor filters to the feature learning stage of CNN. They performed the analysis on ROIs from a composed large mammogram dataset to classify malignant, benign and normal mammograms. They reported the efficiency and application without standardization of the medical images for diagnosis of breast cancer [22]. Touahri et al. suggested a cancer lesion detection based CADx system to identify malignant and benign. They experimented on the various batch normalization techniques on CNN. They highlighted the performance of using local binary patterns with $\mathrm{CNN}$ as the best performance for tumour identification [23].

Depth of the CNN architectures reveals the idea of modelling mobile and pruned CNN architectures with better achievements. This paper aims at proposing a CNN-based cancer classification CADx model with simple architectures with minimized preprocessing and comparing the classification performances with extensive pre-trained architectures.

The remaining of the paper is organized as follows: Digital Database for Screening Mammography (DDSM) and CNN architecture are detailed in Section 2, experimental setup and the achievements are presented in Section 3 and compared to the popular pre-trained CNN architectures. The state-of-the-art and comparison of the classification performances considering independent test metrics, advantages of the proposed CNN model are discussed in Section 4.

\section{Materials and Method}

\subsection{Digital Database for Screening Mammography}

DDSM is a comprehensive mammogram database which was established as a collaborative project of many foremost medicine and medical engineering universities [24]. DDSM consists of a total number of 2620 cases with normal, cancer, and benign classes. The mammograms were scanned by the medical devices (DBA, LUMYSIS, and HOWTEK) with different resolutions. Each case has mediolateral oblique (MLO) and the craniocaudal (CC) views for both left and right breasts. We used CC views in the analysis since $\mathrm{CC}$ provides visualizing most breast tissue without pectoral muscle representations in mammogram. The variety of mammograms from different devices is a preferable characteristic of DDSM for analysis of different image resolutions using DL algorithms.

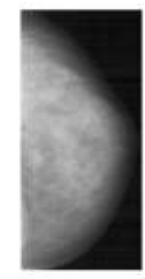

(a)

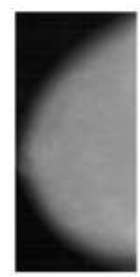

(b)

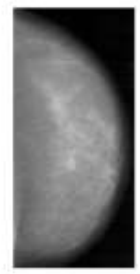

(c)

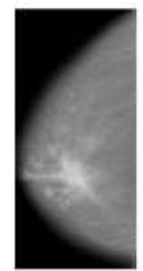

(d)
Fig. 1. The random mammograms from DDSM with normal left breast (a), normal right breast (b), left breast with cancer (c), and right breast with cancer $(\mathrm{d})$

Table 1. Mammogram distribution by cases and scanners in the analysis

\begin{tabular}{lll}
\hline Device & Cancer & Normal (Left and Right) \\
\hline DBA & 97 & $430 \times 2$ \\
Howtek & 424 & $183 \times 2$ \\
Lumysis & 393 & $82 \times 2$ \\
Total & 914 & 1390 \\
\hline
\end{tabular}

We excluded the mammograms with benign from the DDSM to classify complete cases with normal and cancer. We analyzed whole mammograms without extracting ROIs of cancer lesions or other pathologies. Each mammogram has a light pectoral muscle representation and additional descriptors in the volume including date, patient age, breast density, and more. Therefore, we cropped the breast regions beginning from muscles to the nipples to contain complete breast form. By this way, we excluded the nonbreast tissues, additional descriptors, and artifacts causing from the patient posture mistake. Table 1 presents the number of mammograms by the scanners in the analysis. The sample mammograms are indicated in Fig 1 . We increased the mammograms by $4 \mathrm{x}$ using flipping-based augmentation (horizontal, vertical, and both) on cropped mammograms.

\subsection{Convolutional Neural Networks}

Owing to big data and detailed supervised learning with many hidden layers and neurons, training DL models is a timeconsuming process. Dropout regularization and the transfer learning in DL models are the main benefits to overcome this issue. Transfer learning is the pre-trained models with distinct databases; however, the model and the pre-trained weights are optimized using various techniques. In a similar way to genetic algorithms, the training starts with a specific space for weights. Moreover, the dropout technique accelerates the training of the fully-connected layers by excluding the neurons in a hidden layer 
by similarity index [8]. CNN is the most popular DL algorithm for classification and pathology detection for medical image analysis. The CNN was supported by various optimizations and unsupervised techniques to learn the representations of the input data on feature activation maps [11]. The main benefits of CNN are robustness to overfitting for large datasets, fluency in detailed analysis of feature maps at each feature learning layer, and using many hidden layers with fully connected neuron structure.

The CNN model is comprised of feature learning stage, flattening layer, and fully connected layers (FC). Feature learning stage consists of different sequences and repetition variations of convolutional layer (CONV), pooling layer, and rectified-linear unit (RELU). The structure of CNN is depicted in Fig. 3. CONV layer outputs different representations of the input image considering filter depth. Whereas pooling layer down-samples the representations by transferring the dominant pixels at a specific filter size, RELU enables outputting non-negative values for pixels [25]. The number of the CONVs enables extracting different representations with feature maps at various levels [26]. Whereas the first CONVs identify low-level features, the latter CONVs attain high-level features by transferring the first activation maps to next layers [25]. Therefore, composing a CNN model has many variations in the depth of model, size of filters, number of hidden layers and neurons, deciding the level of the learned features according to classes, and more [27]. The last representation of the feature learning stage is converted to a feature set array in flattening layer for feeding the fully connected layers as input.

The RELU activation function is defined as follows:

$$
f_{R E L U}\left(x_{i}\right)=\max \left(0, x_{i}\right)
$$

where $x_{i}$ represents for $i^{\text {th }}$ pixel of feature map $x$ after CONV. Sequentially, FC layer calculates the neuron weights using:

$h_{W, b}(\hat{x})=f\left(W^{T} \widehat{x}_{\iota}+b\right)$

where $W, b$, and $\hat{x}$ are weights, bias, and input of $h_{w, b}$ neuron in FC. $f(\cdot)$ represents for the activation function of the neurons in hidden layer.

\section{Experimental Results}

The mammograms should be analyzed, meticulously, since they contain pectoral muscle tissues, additional information about patients, patient pose faults, and device-based noises. Moreover, cancer lesions at early stages may be easily confused with noises from the device. Therefore, we experimented on various $\mathrm{CNN}$ architectures and supervised structures for enhancing the performance of proposals to classify the mammograms normal or cancer. In this study, we shared the best achievements for CNN architectures and performed a complete comparison with the existing literature on DDSM.

Cancer and normal mammograms were pre-processed before the analysis. We cropped the whole breast tissue by excluding muscle and additional information on the mammograms. Data augmentation was performed on the mammograms to learn various representations for cancer and normal cases and to avoid overfitting. The mammogram database was increased by 4 times (original + flipping vertically, horizontally, and both). The details of the data augmentation process on DDSM are depicted in Fig. 2. Using this augmentation procedure, we got 914 and 1390 mammograms for cancer and normal cases, sequentially. Each mammogram was resized to $224 \times 224$ pixels to constitute a standardized CNN input.

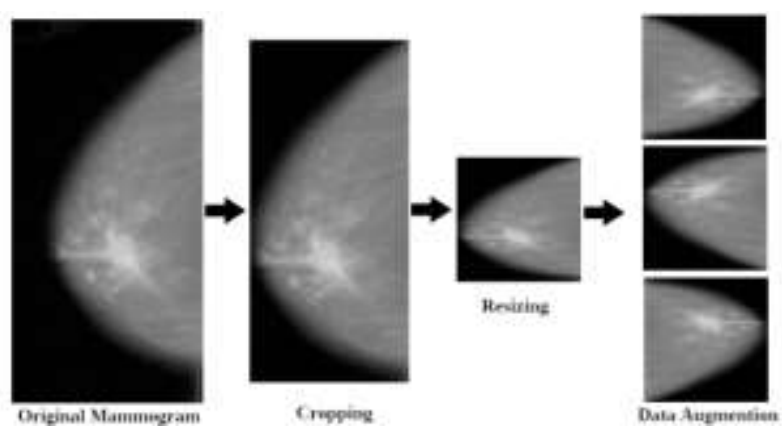

Fig. 2. Pre-processing of mammograms.

Hereby, experiments were carried out on various CNN architectures using the adaptability of DL algorithms to medical images. The conventional CNN architectures with FCs were trained using 10-fold cross validation. In this validation method, the database was folded into ten parts. Whereas the nine folds merged to train the proposed $\mathrm{CNN}$ architectures, the remaining fold was used to test the trained model. The validation is terminated when each fold is utilized as a testing fold, separately. The average of performance of ten testing folds is presented as the evaluation value of CNN architectures. Accuracy, sensitivity, and specificity performance metrics were calculated as independent test characteristics for objective assessment of the CNN architectures [28, 29].

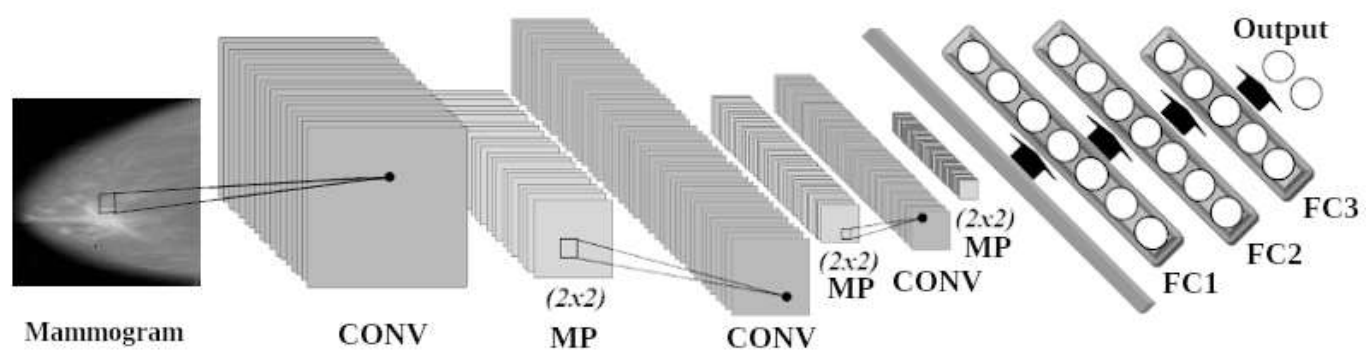

Fig. 3. The proposed Convolutional Neural Networks architecture layer-by-layer 
Table 2. The best five classification performances (\%) for CNN models with fully-connected layers.

\begin{tabular}{|c|c|c|c|}
\hline CNN Model & $A C C$ & SEN & $S P E$ \\
\hline \multicolumn{4}{|l|}{ CONV(3)@96,CONV(3)@64,MP,RELU } \\
\hline CONV(6)@64,CONV(5)@32,MP,RELU & 88.59 & 93.22 & 95.04 \\
\hline \multicolumn{4}{|l|}{ CONV(6)@32,MP,RELU } \\
\hline \multicolumn{4}{|l|}{ CONV(5)@96,MP,RELU } \\
\hline CONV(8)@64,CONV(4)@64,MP,RELU & 90.19 & 96.17 & 97.16 \\
\hline \multicolumn{4}{|l|}{ CONV(8)@96, MP, RELU } \\
\hline \multicolumn{4}{|l|}{ CONV(5)@96,CONV(7)@32, MP,RELU } \\
\hline CONV(6)@64,MP,RELU & 91.02 & 95.84 & 96.98 \\
\hline \multicolumn{4}{|l|}{ CONV(7)@32,CONV(4)@64,MP,RELU } \\
\hline \multicolumn{4}{|l|}{ CONV(7)@96, MP, RELU } \\
\hline CONV(8)@64,CONV(3)@32, MP, RELU & 91.62 & 98.91 & 99.18 \\
\hline \multicolumn{4}{|l|}{ CONV(3)@96, MP,RELU } \\
\hline \multicolumn{4}{|l|}{ CONV(3)@64,CONV(4)@64,MP,RELU } \\
\hline CONV(2)@64,MP,RELU & 92.84 & 95.30 & 96.72 \\
\hline CONV(5)@96, MP, RELU & & & \\
\hline
\end{tabular}

FC: Fully-connected, MP: Max pooling, SEN: Sensitivity, ACC: Accuracy, SPE: Specificity

In the feature learning stage of the proposed $\mathrm{CNN}$ architectures, the CONV filter size, numbers of filters were iterated at certain ranges. Max-pooling with a size of $2 \times 2$ was applied to down-sample the feature maps and was followed by a RELU. The depth of CONVs was fixed at three. The filter size for CONVs was randomly selected between 3 8. We established the CNN architectures with a fixed supervised training to assess the effect of feature learning on learning procedure for mammogram classification. We used three FCs with fixed neuron numbers (100 neurons, 100 neurons, and 50 neurons, respectively). The same classification parameters including learning rate of 0.001 , dropout factor of $0.5,200$ epochs, and Adam optimization were set for training of FCs in CNN proposals. The output function of the CNN was softmax with mean square error loss function.

The best classification performances for breast cancer are presented in Table 2 and are depicted in Fig. 4. Experimental results show that $\mathrm{CNN}$ can separate mammograms into normal and cancer for breast cancer using simple architectures. CNN models with small filter sizes and increasing number of filters layer-by-layer had better generalization performances. Using 32 filters at the first CONV and 96 filters at the last CONV reached the best achievements due to analysis of more activation feature maps and transferring them to the supervised learning. The highest classification performance was achieved with the rates of $92.84 \%, 95.30 \%$, and $96.72 \%$ for overall accuracy, sensitivity, and specificity, respectively. The feature learning stage of CNN model was sequenced using the layers including two CONVs with 64 filters, max pooling, a CONV with 64 filters, max pooling, CONV with 96 filters, max pooling, and flatten layer.

\section{Discussion}

The CADx systems on mammograms for breast cancer classification have up to date developments using novel approaches on medical image analysis. DL algorithms including generative and classification models provide reliable, robust and detailed analysis on separation of cancer lesions from healthy breast tissue. The analysis capability of feature leaning phase of $\mathrm{CNN}$ provides a more enhanced and responsible feature extraction and transfer between layers for CADx systems. DL has advantages of minimizing pre-process and excluding feature extraction form the medical analysis. This study aimed at experimenting with $\mathrm{CNN}$ architectures with a fixed supervised training to evaluate the impact of CONVs on mammograms for breast cancer.

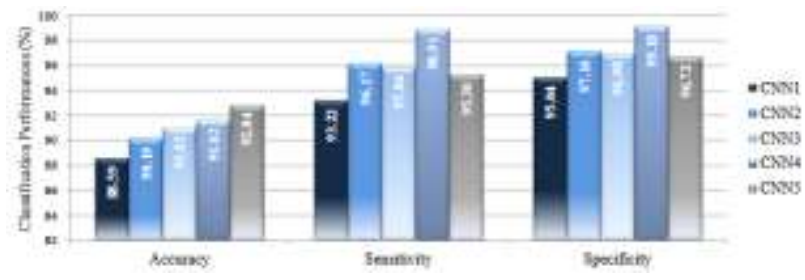

Fig. 4. Comparison of proposed Deep Learning architectures considering system performances $(\%)$

A majority of novel researches applied the pre-trained popular $\mathrm{CNN}$ architectures to classify mammograms for breast cancer. Especially, transfer-learning approach is the most common approach with pre-trained weights due to fast optimization and adaptability abilities of CNN. The state-of-art of CNN researches for classification of cancer and normal mammograms on DDSM are presented in Table 3.

Pardamean et al. applied transfer learning on ChexNet model for breast cancer. They utilized DenseNet architecture for feature learning stage and iterated on various supervised models using many FCs, dropout factor and other learning parameters. They reached a separation accuracy rate of $90.38 \%$ [19]. Suzuki et al. also applied transfer learning on ImageNet weights. They utilized AlexNet architecture for feature learning and re-trained the model on mammograms. Their highest achievements are accuracy rate of $85.35 \%$ and sensitivity rate of $89.90 \%$ [21]. Nasir Khan et al. utilized VGGNet, GoogleNet, and ResNet architectures on multi-view feature fusion model. They performed binary classifications (cancernormal, mass-calcification, and malignant-benign). They highlighted the best performance on classification of cancernormal mammograms as VGGNet with accuracy rate of $94.45 \%$, sensitivity rate of $98.07 \%$, and specificity rate of $88.13 \%$ [30]. Swiderski et al. proposed own CNN architecture using the non-negative matrix factorization method at the feature learning stage. They separated cancer and normal mammograms with an accuracy rate of $85.82 \%$, a sensitivity rate of $82.38 \%$, and a specificity rate of $86.59 \%$ [31].

Whereas the most of the researches on CNN utilized pretrained CNN models, this paper proposed own CNN architecture on mammograms. The proposals aimed at defining the most responsible feature activation map using sequential CONVs. The achievements produced that in the iterated range of CONV variations simplistic models with sequentially convolved with big number of filters at the last CONV provided learning more responsible characteristics on 
Table 3. The state-of-art for CNN architectures on mammograms for breast cancer detection on DDSM.

\begin{tabular}{lllccc}
\hline Related Studies & \multicolumn{1}{c}{ Methods } & Architecture & Accuracy & Sensitivity & Specificity \\
\hline Nasir Khan et al. & Multi-View Feature Fusion & VGGNet & 94.45 & 98.07 & 88.13 \\
Pardamean et al. & Transfer learning (ChexNet) & DenseNet & 90.38 & - & - \\
Swiderski et al. & Non-negative matrix factorization & CNN & 85.82 & 82.38 & 86.59 \\
Suzuki et al. & Transfer learning (ImageNet) & AlexNet & 85.35 & 89.90 & - \\
This study & Cropping & CNN & 92.84 & 95.30 & 96.72 \\
\hline
\end{tabular}

mammograms for breast cancer. The proposed CNN architecture with the highest classification performance reached the rates of $92.84 \%, 95.30 \%$, and $96.72 \%$ for accuracy, sensitivity, and specificity, respectively. Using sequential CONVs before max-pooling enabled analysis of mammograms within various representations of the feature maps. The best $\mathrm{CNN}$ architecture in the proposals is established by $\operatorname{CONV}(3 \times 3)$ with 32 filters, $\operatorname{CONV}(5 \times 5)$ with 64 filters, maxpooling, RELU, CONV(8x8) with 96 filters, max-pooling, RELU , FC1(100), FC2(100) and FC3(50), respectively. It is noted that each max-pooling layer has a filter size of $2 \times 2$ for down sampling; CONVs have no-stride and no padding.

\section{Conclusion}

We explored the impact of various feature-learning stages in $\mathrm{CNN}$ on CONV and pooling layer variations. The existing literature applied transfer-learning approaches on pre-trained $\mathrm{CNN}$ architectures; however, these models have a big number of classification parameters considering the depth of feature learning and supervised stage. The paper contributed on the idea that simple pruned $\mathrm{CNN}$ architectures have ability to classify mammograms into cancer and normal cases on mammograms with a detailed feature-learning phase. The proposed CNN architecture provides integrating Deep Learning models into mobile networks and real-time devices for analysis of medical images.

\section{References}

[1] E. A. Sickles, "Screening for breast cancer with mammography," Clinical Imaging. 1991, doi: 10.1016/s01406736(01)07198-7.

[2] A. Jalalian, S. B. T. Mashohor, H. R. Mahmud, M. I. B. Saripan, A. R. B. Ramli, and B. Karasfi, "Computer-aided detection/diagnosis of breast cancer in mammography and ultrasound: a review," Clin. Imaging, vol. 37, no. 3, pp. 420426, May 2013, doi: 10.1016/j.clinimag.2012.09.024.

[3] Q. Zeng, H. Jiang, and L. Ma, "Learning multi-level features for breast mass detection," in ACM International Conference Proceeding Series, 2018, doi: 10.1145/3285996.3286000.

[4] H. P. Chan, R. K. Samala, L. M. Hadjiiski, and C. Zhou, "Deep Learning in Medical Image Analysis," in Advances in Experimental Medicine and Biology, 2020.

[5] J. Shiraishi, Q. Li, D. Appelbaum, and K. Doi, "Computer-aided diagnosis and artificial intelligence in clinical imaging," Seminars in Nuclear Medicine. 2011, doi: 10.1053/j.semnuclmed.2011.06.004.

[6] M. A. Al-antari et al., "An Automatic Computer-Aided Diagnosis System for Breast Cancer in Digital Mammograms via Deep Belief Network," J. Med. Biol. Eng., 2018, doi: 10.1007/s40846-017-0321-6.
[7] S. J. A. Sarosa, F. Utaminingrum, and F. A. Bachtiar, "Mammogram Breast Cancer Classification Using Gray-Level Co-Occurrence Matrix and Support Vector Machine," in 3rd International Conference on Sustainable Information Engineering and Technology, SIET 2018 - Proceedings, 2018, doi: 10.1109/SIET.2018.8693146.

[8] R. Wang et al., "Multi-level nested pyramid network for mass segmentation in mammograms," Neurocomputing, vol. 363, pp. 313-320, Oct. 2019, doi: 10.1016/j.neucom.2019.06.045.

[9] W. Zhu, X. Xiang, T. D. Tran, G. D. Hager, and X. Xie, "Adversarial deep structured nets for mass segmentation from mammograms," in 2018 IEEE 15th International Symposium on Biomedical Imaging (ISBI 2018), 2018, pp. 847-850, doi: 10.1109/ISBI.2018.8363704.

[10] G. Altan and Y. Kutlu, "Hessenberg Elm Autoencoder Kernel For Deep Learning," J. Eng. Technol. Appl. Sci., 2018, doi: 10.30931/jetas.450252.

[11] G. Altan, Y. Kutlu, A. Ö. Pekmezci, and A. Yayık, "Diagnosis of Chronic Obstructive Pulmonary Disease using Deep Extreme Learning Machines with LU Autoencoder Kernel," in 7th International Conference on Advanced Technologies (ICAT'18), 2018.

[12] D. Abdelhafiz, C. Yang, R. Ammar, and S. Nabavi, "Deep convolutional neural networks for mammography: Advances, challenges and applications," BMC Bioinformatics, 2019, doi: 10.1186/s12859-019-2823-4.

[13] K. Mendel, H. Li, D. Sheth, and M. Giger, "Transfer Learning From Convolutional Neural Networks for Computer-Aided Diagnosis: A Comparison of Digital Breast Tomosynthesis and Full-Field Digital Mammography," Acad. Radiol., vol. 26, no. 6, pp. 735-743, Jun. 2019, doi: 10.1016/j.acra.2018.06.019.

[14] J. Arevalo, A. Cruz-Roa, and F. A. González, "Hybrid image representation learning model with invariant features for basal cell carcinoma detection,” 2013, pp. 89220M--6, doi: 10.1117/12.2035530.

[15] J. Arevalo et al., "DeepMammo Breast Mass Classification using Deep Convolutional Neural Networks," Comput. Methods Programs Biomed., 2018, doi: 10.1016/j.acra.2018.06.019.

[16] Pan, J. Zhang, T. Wang, and L. Kong, "X-Ray Mammary Image Segmentation Based on Convolutional Neural Network," in 2019 IEEE 4th International Conference on Image, Vision and Computing (ICIVC), 2019, pp. 105-108, doi: 10.1109/ICIVC47709.2019.8981350.

[17] Yoon and S. Kim, "AdaBoost-based multiple SVM-RFE for classification of mammograms in DDSM," BMC Med. Inform. Decis. Mak., 2009, doi: 10.1186/1472-6947-9-S1-S1.

[18] P. Xi, C. Shu, and R. Goubran, "Abnormality Detection in Mammography using Deep Convolutional Neural Networks," in 2018 IEEE International Symposium on Medical Measurements and Applications (MeMeA), 2018, pp. 1-6, doi: 10.1109/MeMeA.2018.8438639. 
[19] B. Pardamean, T. W. Cenggoro, R. Rahutomo, A. Budiarto, and E. K. Karuppiah, "Transfer Learning from Chest X-Ray Pretrained Convolutional Neural Network for Learning Mammogram Data," Procedia Comput. Sci., vol. 135, pp. 400407, 2018, doi: 10.1016/j.procs.2018.08.190.

[20] M. G. Ertosun and D. L. Rubin, "Probabilistic visual search for masses within mammography images using deep learning," in 2015 IEEE International Conference on Bioinformatics and Biomedicine (BIBM), 2015, pp. 1310-1315, doi: 10.1109/BIBM.2015.7359868.

[21] S. Suzuki et al., "Mass detection using deep convolutional neural network for mammographic computer-aided diagnosis," in 2016 55th Annual Conference of the Society of Instrument and Control Engineers of Japan (SICE), 2016, pp. 1382-1386, doi: 10.1109/SICE.2016.7749265.

[22] V. D. Nguyen, K. Lim, M. D. Le, and N. Dung Bui, "Combination of Gabor Filter and Convolutional Neural Network for Suspicious Mass Classification," in 2018 22nd International Computer Science and Engineering Conference (ICSEC), 2018, pp. 1-4, doi: 10.1109/ICSEC.2018.8712796.

[23] R. Touahri, N. AzizI, N. E. Hammami, M. Aldwairi, and F. Benaida, "Automated Breast Tumor Diagnosis Using Local Binary Patterns (LBP) Based on Deep Learning Classification," in 2019 International Conference on Computer and Information Sciences (ICCIS), 2019, pp. $1-5, \quad$ doi: 10.1109/ICCISci.2019.8716428.

[24] R. M. and P. K. J. M. Heath, K. Bowyer, D. Kopans, "The Digital Database for Screening Mammography," in the Fifth International Workshop on Digital Mammography, M.J. Yaffe, ed., Medical Physics Publishing, 2001., 2001, doi: ISBN 1930524-00-5.

[25] A. Krizhevsky, I. Sutskever, and G. E. Hinton, "ImageNet Classification with Deep Convolutional Neural Networks," Adv. Neural Inf. Process. Syst., 2012, doi: http://dx.doi.org/10.1016/j.protcy.2014.09.007.

[26] D. C. Cireşan, U. Meier, L. M. Gambardella, and J. Schmidhuber, "Convolutional neural network committees for handwritten character classification," in Proceedings of the International Conference on Document Analysis and Recognition, ICDAR, 2011, doi: 10.1109/ICDAR.2011.229.

[27] G. Altan, "DeepGraphNet: Grafiklerin Sinıflandırılmasında Derin Öğrenme Modelleri,” Eur. J. Sci. Technol., pp. 319-327, Oct. 2019, doi: 10.31590/ejosat.638256.

[28] G. Altan and Y. Kutlu, "Generative Autoencoder Kernels on Deep Learning for Brain Activity Analysis," Nat. Eng. Sci., 2018, doi: 10.28978/nesciences.468978.

[29] G. Altan, Y. Kutlu, and N. Allahverdi, "Deep Learning on Computerized Analysis of Chronic Obstructive Pulmonary Disease," IEEE J. Biomed. Heal. Informatics, vol. 24, no. 5, pp. 1344-1350, May 2020, doi: 10.1109/JBHI.2019.2931395.

[30] H. Nasir Khan, A. R. Shahid, B. Raza, A. H. Dar, and H. Alquhayz, "Multi-View Feature Fusion Based Four Views Model for Mammogram Classification Using Convolutional Neural Network," IEEE Access, 2019, doi: 10.1109/ACCESS.2019.2953318.

[31] B. Swiderski, J. Kurek, S. Osowski, M. Kruk, and W. Barhoumi, "Deep learning and non-negative matrix factorization in recognition of mammograms," in Eighth International Conference on Graphic and Image Processing (ICGIP 2016), 2017, doi: 10.1117/12.2266335. 